\title{
Tension-Free Vaginal Tape For Treating Stress Urinary Incontinence ( Types I And Ii ) : Experience With 32 Cases.
}

\author{
*M. H. El-Shazly, and **Nagwa A. Ghaffar. \\ *Urology Department; El-Sahel Teaching Hospital; \\ **Ob/Gyn Department, Al Azhar University (Girls), Cairo, Egypt.
}

\begin{abstract}
:
Objectives: To evaluate the clinical outcome of using tension-free vaginal tape (TVT) for treating types I and II genuine stress urinary incontinence. Patients and Methods: The study comprised a total of 32 women with genuine stress urinary incontinence types I and II treated with TVT between 1999 and 2002. The mean follow-up period was 19.4 months. Results: The success rate was $87.5 \%(28 / 32$ patients). The cure rate was durable in the majority of successful cases $26 / 28(92.9 \%)$ during the one year follow-up period except for $2 / 28(7.1 \%)$ women in whom stress urinary incontinence recurred in a milder form. Postoperative retention of urine occurred in 2/32 (6.3\%) patients, which was transient and responded well to urethral dilatation under local anaesthesia. Details of the clinical outcome variables and complications are represented. Conclusion: The TVT procedure is an effective and minimally invasive alternative to surgical options for managing types I and II stress urinary incontinence. The complication rates are low and hospital stay is short and the procedure is feasible under local anaesthesia.
\end{abstract}

Keywords: stress incontinence, TVT, tension-free vaginal tape.

\section{Introduction:}

Tension-free vaginal tape (TVT) is a relatively new minimally invasive procedure for managing stress urinary incontinence which gained a wide acceptance by both urologists and gynecologists during the last decade with high success rates, low morbidity and complication rates [6]. Moreover, the hospital stay is short and the procedure can be done as a day-case under local anaesthesia. The standard procedure implicates the use of a synthetic polypropylene mesh sling placed in the mid-urethral position [4]. However, further randomized controlled comparative studies between the TVT procedure and the gold standard colposuspension options are to be awaited as well as its long term results.

\section{Aim of the work:}

To assess the effectiveness and clinical outcome of the TVT procedure in treating types I and II stress urinary incontinence according to the Urodynamics Society classification in 1997 [20]

\section{Patients and methods:}

Between 1999 and 2002, a total of 32 women (mean age 53.6 years, range 37-64 years) with urodyna -micallyproved types I and II genuine stress urinary incontinence were treated with the TVT procedure,

and followed-up for one year at minimum, in two centers: Urology Department, El-Sahel Teaching48 Hospital, Cairo, Egypt and Urology and 
Obs/Gyn Departments, Mouwasat Hospital, Dammam, Saudi Arabia.

The study included only types I and II SUI who failed to respond to other medical conservative options. All cases with type III SUI (intrinsic sphincteric deficiency) or mixed SUI (detrusor overactivity + SUI) were excluded. In all cases, the TVT procedure was the primary treatment option with no case of previous surgical management for stress urinary incont inence. Cases with previous major pelvic surgeries like abdominal or vaginal hysterectomy were excluded.

The preoperative work-up included a detailed medical history, physical examination, gynecologic examination, abdominopelvic ultraso und with estimation of post-voiding residual volume. The urodynamic asse ssment protocol included uroflowmetry, filling cystometry, and urethral pressure profile at rest and during stress record ing the leak point pressure $\left(\right.$ Duet $^{\circledR}$ Multi-P, Medtronic, Denmark). The diagnosis of stress urinary incontinence was made whenever urine leakage occurred during coughing or straining with no associated detrusor activity. The filling rate was $50 \mathrm{ml} / \mathrm{min}$ with the patient in a semi-sitting position. The same urodynamic evaluation was repeated for all cases 6 and 12 months after the TVT procedure. Subjectively, patient's satisfaction and quality of life (QoL) was post-operatively monitored as cured, markedly improved, impr oved, no change, or worse in patient's terms besides an enquiry about the voiding pattern regarding hesitancy, sense of incomplete emptying, straining to void, or urgency. Our urodynamic follow-up criteria define outflow obstruction if $\mathrm{Q}_{\max }$ is $\leq 12 \mathrm{ml} / \mathrm{s}$ and significant post-voiding residual volume if $\geq 25 \%$ of the bladder capacity.
Our follow-up protocol included regular outpatient clinic visits at 4 weeks, and months 3, 6, 9, and 12. After 12 months the patients were instructed to attend the outpatient clinic in case of any urological complaint or yearly for longer-term follow-up. In each visit subjective assessment of patient's satisfaction, clinical examination, urin alysis, and pelvic ultrasound examin ation with full bladder and post-voiding were done.

Spinal anaesthesia was used in $12 / 32 \quad(37.5 \%)$ patients, epidural anaesthesia in $9 / 32(28.1 \%)$ patients, and local infiltration anaesthesia using an average dose of $40 \mathrm{ml}$ of lidocaine $2 \%$ in $11 / 32(34.4 \%)$ patients. The surgical technique followed the original one described by Ulmsten et al in 1996 [20]. The tape used is a synthetic polypropylene mesh tape (Ethicon Gynecare $^{\mathrm{TM}}$, UK ).

Under local, spinal, or epidural anaesthesia a midline anterior vaginal wall incision is made over the urethra with 18 Fr Foley's catheter in situ to facilitate urethra-bladder neck orient ation. Creation of two para-urethral tunnels is then performed and the two TVT needles are then passed paraurethrally at a mid-urethral position and directed posterior to the symphysis pubis to come out through two separates small suprapubic incisions. Each needle-path is checked endoscopically to avoid and detect any bladder or urethral perforations. Then, both needles and the tape plastic covers are removed without exerting any tension on the tape. An on-table stress test is done through asking the patient to cough or strain with a bladder contai ning 250-300 $\mathrm{ml}$ of normal saline. Postoperatively, we did not fix urethral catheter routinely unless a bladder or urethral perforation has occurred or excessive dissections were performed. 
This occurred in 2/32 (6.3\%) patients where a urethral catheter was kept for 5 days and both of them voided freely after catheter removal.

\section{Results:}

Table (1) summarizes the patients, procedural, and clinical outc ome data. In this study, the follow-up protocol was 12 months at minimum, with a mean follow-up period of 19.4 (range 12-50) months. Out of the 32 women, 29 patients $(90.6 \%)$ completed the 12 month follow-up protocol except for two women who did not show at $9^{\text {th }}$ and $12^{\text {th }}$ month visits because they feel cured as explained by phone contact. Another women did not come after the month-3 visit (where she declared cure) and could not be contacted later. The mean hospital stay was 1.2 days (range 12-72 hours) with most of the cases done as day cases (25/32 "78.1\%"). The mean operative time was 25.6 minutes (range 20-54 minutes).

The procedure is considered successful if the patient declared being cured, markedly improved, or impro ved. Our success rate was $87.5 \%$ (28/32). Out of the 28 women $21 / 32$ $(65.6 \%)$ declared complete cure, $5 / 32$ $(15.6 \%)$ markedly improved, and $2 / 32$ (6.3\%) improved. The procedure was not successful in 4/32 (12.5\%) women in whom no change was declared with no case getting worse. The successful outcome was durable throughout the one year follow-up period in 26/28 women $(92.9 \%)$ with $2 / 28$ women $(7.1 \%)$ of recurring stress urinary incontinence starting gradually after an average of 6.4 months postoperatively. In both patients the recurring stress urinary incontinence was lesser in severity and did not progress further during the 12 months post-operative follow-up period.

Post-operative retention of urine occurred in $2 / 32(6.3 \%)$ patients, and was managed by urethral dilatation under local lidocaine $2 \%$ gel up to 26 Fr. with the intention of stretching the tape and forcing the straight metal dilator dorsally to decrease the tape tightness. Both women improved after urethral dilatation and no significant post-voiding residual urine was detected in the follow-up period. Newly develo ped urge sensation with urodynami cally-proved detrusor hyperactivity was documented in $3 / 32(9.4 \%)$ patients which was transient (for a mean of 10.5 months) in all the three cases and responded well to oxybutinin chloride 5 mg tablets 2-3 times daily. In 4/32 $(12.5 \%)$ patients, urinary tract infection was documented and all responded well to appropriate antibiotic therapy. One case $(3.1 \%)$ had mild vaginal wound infection with no wound dehiscence. In no case bladder perforation, urinary retention, or urethral erosion had occurred. 
Table [1] Patients, Procedural, and Clinical Outcome Data.

Age

Follow-up Period.

Anaesthesia:

- Local

- Spinal

- Epidural

Operative Time

Hospital Stay:
Mean, 53.6 years.

Mean, 19.4 months.

11/32 (34.4\%) patients.

$12 / 32(37.5 \%)$ patients.

9/32 (28.1\%) patients.

Mean 25.6 minutes.

Mean, 1.2 days.

25/32 (78.1\%) as Day Cases.
Range, 36-64 years. Range, 12-50 months.

Range 20-54 min. Range 12-72 hours.

Success Rates:

- Complete Symptom Cure

- Marked Improvement.

- Improved.

Failure Rates:

- No Improvement.

- Getting Worse.

Success Durability at One Year:

Complications:

- Recurrent SUI ( milder)

- Transient Retention.

- Transient de novo Urge

- Bladder Perforation

- Urinary Tract Infection

- Mild Wound Infection
21/32 (65.6\%) patients.

$5 / 32(15.6 \%)$ patients.

2/32 (6.3\%) patients.

Overall 28/32(87.5\%) patients.

4/32 (12.5\%) patients.

$0 / 32(00.0 \%)$ patients.

26/28 (92.9\%) patients.

$2 / 28(7.1 \%)$ patients.

2/32 $(6.3 \%)$ patients.

$3 / 32(9.4 \%)$ patients.

$2 / 32(6.3 \%)$ patients.

$4 / 32(12.5 \%)$ patients.

$1 / 32(3.1 \%)$ patients.

\section{Discussion:}

Challenging the pressure-transm ission theory, the more recent integral theory of female continence stated that stress urinary incontinence in females results from failure of the active closure mechanism due to laxity of the pubour ethral ligament $[8,16]$. Urodynamically, genuine SUI is defined as involuntary urinary leakage with increased intraabdominal pressure without associated detrusor contract -ions. According to the classification of Blaivas, in types I and II the urinary leakage is due to urethral hypermobility without intrinsic urethral sphincteric deficiency [4]. In both, the baldder neck is closed at rest and urine leaks only during straining associated with urethral decent below the inferior margin of symphysis pubis. However, in type $I$, the decent is less than 2 centimeters and there is no or minimal associated cystocele. In type II, the cystocele is more evident and the urethral decent is more than $2 \mathrm{~cm}$. In type III, the bladder neck is open at rest with maximum urethral closure pressure less than $20 \mathrm{~cm}$ water. The urine leaks with no or minimal stress and even by gravity due to deficient intrinsic sphincteric activity with no urethral 
hypermobility. In mixed SUI, the genuine SUI is associated with detrusor overactivity or hyper-reflexia.

Although the TVT procedure elevates bladder neck to a much lesser extent than colposuspensions, it mainly acts through inducing a urethral kink at tape level (mid-urethral) during stress which augments the urethral pressure substituting for the hammock-like effect of a normal pubourethral ligament [1]. Historically, pubovaginal slings for treating stress urinary incontinence started almost a century ago [10]. Colposuspensions and pubovaginal slings passed the time test, proved efficacy with durable effect and high success rates and constituted the goldstandard options for managing the three types of stress urinary incontinence $[6,9,10,13]$. In comparison to the TVT procedure, colposuspensions have higher morbidity and complication rates. The hospital stay is longer and the bladder outlet obstructive sequelae are higher [6].

The overall reported success rate of TVT in multicenter studies including 1339 cases with a mean follow-up period of 21 months, is $88 \%$ objective cure rate with $9 \%$ of further subjective improvement [6,21]. Our success rate $28 / 32(87.5 \%)$ is in consistence with most of the reported studies using the same technique [11,13-15,17-19]. We did not include type III stress urinary incontinence "intrinsic sphincteric deficiency" in this study. However, few other studies reported a mean of $75 \%$ success rate in using TVT for type III stress urinary incontinence $[3,12,13]$. Several studies included both genuine and recurrent stress urinary inconti nence for TVT procedure. The treat ment outcomes of TVT in women as a primary procedure appear marginally better than in patients in whom previous surgery for urinary incontinence has failed $[6,11]$.

The reported common morbidity and complications of the TVT procedure include bladder perforation, haematomas, urinary tract infections, wound infections, wound dehiscence, urethral erosion, de novo urge incontinence, and bladder outlet obstru ctive sequelae. The overall reported mean complication rate of the TVT procedure is $6 \%$ with de novo urge incontinence or persistent urge as the most common and most troublesome one $(15 \%)$ and urethral erosion the most serious [15,18]. In our study the complication rates are compatible with those reported previously $[6,15,16]$. Transient post-operative urinary retent ion occurred in 2/32 (6.3\%) women. De novo urgency due urodynamicallyproven detrusor hyperactivity was recorded in $3 / 32(9.4 \%)$ women. In all the three cases, the urge was transient, not associated with significant postvoiding residual volume increase, and effectively controlled by oxybutinin chloride $5 \mathrm{mg}$ tablets 2-3 times daily. A recent randomized controlled study concluded that obstructive voiding dysfunctions including detrusor hyper activity are similar after TVT and colposuspensions [13]. However, Atherton and Stanton concluded that TVT may be even more obstructive than colposuspension and that although TVT procedure has been introduced as "a tension-free vaginal tape" the sling can have tension applied by the surgeon [2]. This indicates the vital technical importance of not exerting any tension on tape positioning which is a common tendency noticed to be shared by most surgeons. Positioning the tape at the bladder neck level and not at the midurethral level is suggested by some that it might be less obstructive [17]. 
However, this awaits further random ized controlled studies to reach a solid conclusion in this concern. Documented urinary tract infections occurred in 4/32 $(12.5 \%)$ women which responded well to appropriate antibiotic therapy in all cases. Overall, the complications reported in our study were mild, transient, and successfully managed conservatively. No general complicat ions were documented in this study. Other rare major complications are reported in the literature like major vascular injuries and bowel perforations with increased risk in patients with previous open pelvic surgeries [6-7].

\section{Conclusion:}

The TVT procedure is a minimally invasive alternative for surgical options in managing uncomplicated stress urinary incontinence with low morbidity and complication rates. It can be done as a day-case under local anaesthesia with a short hospital stay. The long term results of the TVT procedure and data of further randomized controlled studies on the use of TVT in managing recurrent and/or mixed stress urinary incontinence are to be awaited.

\section{References:}

1 Atherton MJ, Stanton SL. A comparison of bladder neck movement and elevation after tension-free vaginal tape and colposuspension. BJOG 2000; 107: 1366-70

2 Atherton MJ, Stanton SL. TVT and colposuspension; comparison and contrasts of possible mechanism. Neurourol Urodynam 2000; 19: 396-8

3 Azam U, Frazer MI, Kozman EL. Tension free vaginal tape (TVT) in women with previously failed incontinence surgery. Int Urogynecol J Pelvic Floor Dysfunct 1999; 10 (Suppl. 1): S138

4 Blaivas JG, Appell RA, Fautl JA, et al. Definition and classification of urinary incontinence:Recommendations of Urodynamics Society. Neurourol Urodyn 1997a; 16: 149-51

5 Blaivas JG, Jacobs BZ. Pubovaginal fascial sling for the treatment of complicated stress urinary incontin ence. J Urol 1991; 145: 1214

6 Boustead GB. The tension-free vagi nal tape for treating stress urinary incontinence. BJU Int 2002; 89: 687693

7 Brink DM. Bowel injury following insertion of tension-free vaginal tape. $S$ Afr Med J 2000; 90:450-2

8 Constantinou CE. Resting and stress urethral pressures as a guide to mechanism of continence. Clin Obstet Gyynecol 1985; 12: 343-7

9 Cross CA, Cespedes RD, McGuire EJ. Our experience with pubov -aginal slings in patients with stress urinary incontinence. J Urol 1998; 159: 1195-8

10 Goebell R. Zur Operativen Besierigung der Angeborenen Incontinencia Vesical. Z Urol Gynak 1910; 187

11 Haab F, Sananes S, Ammarenco G et al. Results of the tension-free vaginal tape procedure for the treatment of type ii stress urinary incontinence at a minimum followup of 1 year. $J$ Urol 2001; 165: 159-62

12 Haab F, Trockman BA, Zimmern PE, Leach GE. Results of pubova ginal sling for the treatment of intrinsic sphincteric deficiency determined by questionnaire analysis. J Urol 1997; $158: 1738-41$

13 Jacquetin B. Use of "TVT" in surgery for female urinary inconti nence. J Gynecol Obstet Biol Reprod (Paris) 2000; 29: 242-7

14 Moran PA, Ward KL, Johnson D, Smirni WE, Hilton P, Bibby J. Tension-free vaginal tape for primary genuine stress incontinence: a twocentre follow-up study. BJU Int 2000; 86: $39-42$

15 Olsson I, Kroon UB. A three-year postoperative evaluation of tension-free vaginal tape. Gynecol Obstet Invest 1999; 48: 267-9 
16 Petros PE, Ulmsten U. An integral theory of female urinary incontin ence. Experimental and clinical consi derations. Acta Obstet Gynecol Scand Suppl 1990; 153: 7-31

17 Sander P, Moller LMA, Rudnicki PM, Lose G. Does the tension-free vaginal tape procedure affect the voiding phase? Pressure-flow studies before and 1 year after surgery. BJU Int 2002; 89: 694-8

18 Ulmsten U, Johnson P, Rezapour M. A three-year follow-up of tension free vaginal tape for surgical treatment of female stress urinary incontine nce. $B r \quad J$ Obstet Gynaecol 1999; 106: $345-50$
19 Ulmsten U, Falconer C, Johnson P et al. A multicenter study of tension-free vaginal tape (TVT) for surgical treatment of stress urinary incont inence. Int Urogynecol J Pelvic Floor Dysfunction 1998; 9: 210-13

20 Ulmsten U, Henriksson L, Johnson P, Varhos G. An ambulatory surgical procedure under local anesthesia for treatment of female urinary incont inence. Int Urogynecol $J$ Pelvic Floor Dysfunc tion 1996; 7: 81-5

21 Wang AC, Lo TS. Tension-free vaginal tape. A minimally invasive solution to stress urinary incontine nce in women. J Reprod Med 1998; 43: 429-34 
علاج السلس البولى الإجهـادى عند السيـدات

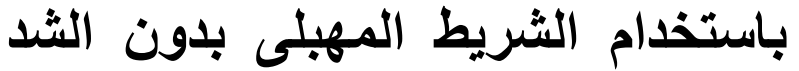

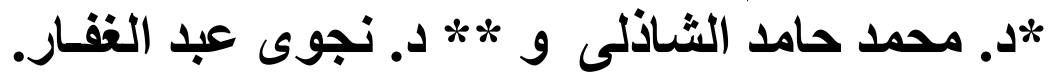

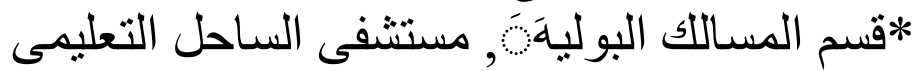

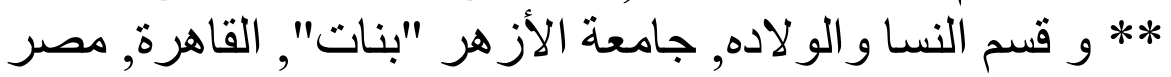

هـدف البحث: تقييم المردود الإكلينيكى لاستخدام الثريط المهبلى بدون الثـد لعلاج السلس البولى الإجهادى الأولى (النوعين 1 و2) عند السيدات.

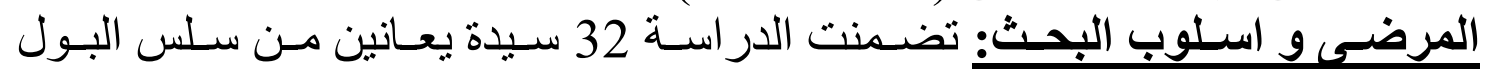

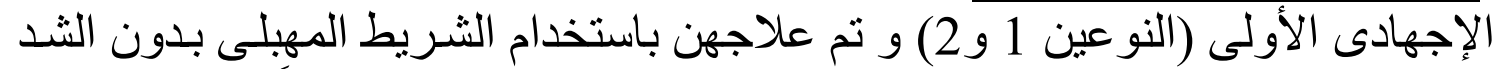

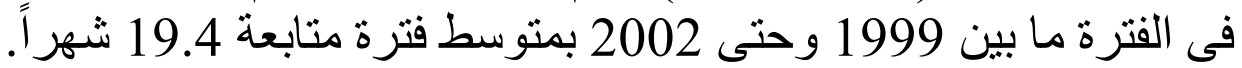
النتـائج: كان معدل الثـفاء

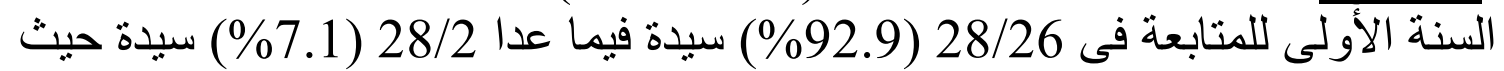

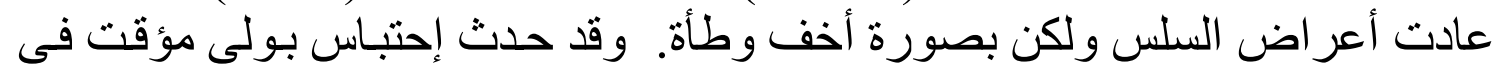

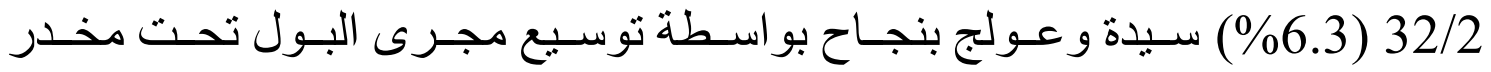

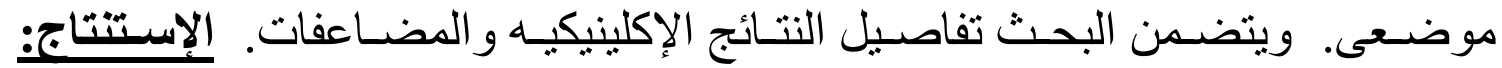

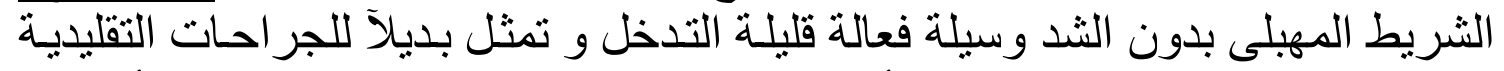

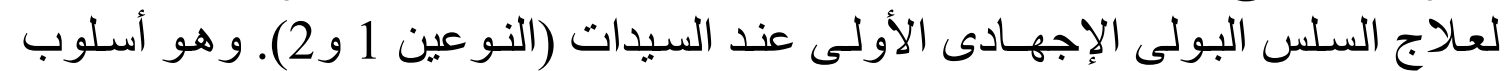

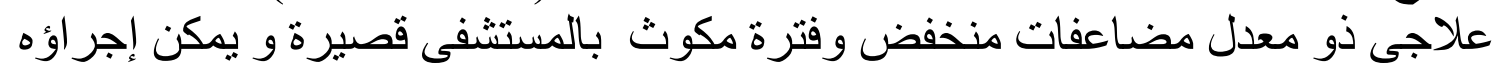
باستخدام التخدير الموضعى. 